Address for Correspondence: Richard A. Chazal, MD, FACC, Valerie Downey, 9800 South HealthPark Drive, Ste 320, Fort Myers, Florida 33908, USA Email: richard.chazal@leememorial.org

\begin{tabular}{|l|}
\hline Access this article online \\
\hline $\begin{array}{l}\text { Website: } \\
\text { www.intern-med.com }\end{array}$ \\
\hline $\begin{array}{l}\text { DOI: } \\
\text { 10.1515/tim-2016-0003 }\end{array}$ \\
\hline Quick Response Code: \\
\\
\\
口) \\
\\
\end{tabular}

\title{
China may hold answers to addressing cardiovascular disease epidemic
}

\author{
Richard A. Chazal \\ President-Elect, American College of Cardiology; Medical Director, Heart and Vascular Institute; Lee Memorial \\ Health System, FL, USA
}

Lessons already learned from the experience in dealing with cardiovascular disease in the United States have the potential for helping China deal with an increasing burden of disease. Lessons that will be learned from the vast patient experiences in China can help shape the face of cardiovascular care worldwide.

Cardiovascular and related diseases are the leading cause of death in countries around the world and China is no exception. According to the World Health Organization (WHO), these diseases are responsible for $45 \%$ of all deaths in China and continue to result in billions of dollars in economic losses. ${ }^{[1]}$ This problem, far from fading, is almost certainly going to become more acute for China, especially in light of disturbing trends in cardiovascular risk factors.

A study published in March 2015 by Yufang $\mathrm{Bi}, \mathrm{MD}, \mathrm{PhD}$, et al., of Shanghai Jiao Tong University School of Medicine, found that only $0.2 \%$ of Chinese men and women have "ideal" cardiovascular health. The authors defined this "as the simultaneous presence of four favorable health behaviors (ideal smoking status, ideal BMI, physical activity at goal, and healthy dietary habits) and four favorable health factors (ideal smoking status, untreated total cholesterol $<200 \mathrm{mg} / \mathrm{dL}$, untreated blood pressure $<120 /<80 \mathrm{~mm} \mathrm{Hg}$, and untreated fasting plasma glucose $<100 \mathrm{mg} / \mathrm{dL}$ ) in the absence of cardiovascular disease history." ${ }^{[2]}$

Rapid economic development in China has influenced adverse trends in lifestyle, including increased intake of saturated fat, physical inactivity, and smoking (especially among men). As a result, Drs. Bi and Ning noted that nearly 3 out of 4 Chinese adults have poor cardiovascular health. ${ }^{[2]}$ The prevalence of diabetes in China is also on the rise, more than doubled over the past decade. Without intervention, the number of Chinese with diabetes is expected to rise to 42.3 million by $2030 .{ }^{[3]}$ Additionally, nearly $42 \%$ of Chinese adult men and women have dyslipidemia. ${ }^{[4]}$ According to investigators from the China National Diabetes and Metabolic Disorders Study, mean levels of total cholesterol, LDL-C, and triglycerides have significantly increased in recent years. Meanwhile, awareness and treatment of dyslipidemia is extremely low. ${ }^{\left[{ }^{[]}\right.}$

The global focus on reducing noncommunicable diseases provides an opportunity for China to take advantage of population-wide strategies to promote cardiovascular health and stop the nearepidemic levels of cardiovascular diseases in the country. The United States and other countries have had success in reducing cardiovascular mortality through patient education and focused campaigns targeting risk factors such as smoking, hyperlipidemia, and hypertension. Cigarette smoking in the U.S. has plummeted from $43 \%$ in 1965 to less than $18 \%$ today, ${ }^{[6]}$ largely due to targeted public education campaigns that educate the public about the impact of smoking on health. Non-smoking laws have also reduced environmental tobacco exposure. Use of clinical data registries and targeted programs focused on closing gaps in the use of clinical guidelines have also played major roles in controlling hypertension and increasing diagnosis and treatment of high cholesterol, diabetes, and other cardiovascular disease risk factors.

Chinese policymakers, physicians, and other stakeholders have an unprecedented opportunity to curb the rise of cardiovascular 
disease. "China is the most populous country in the world, and changes in disease prevalence in the Chinese population will significantly affect the global burden of disease," wrote Dr. Bi and colleagues. ${ }^{[2]}$ It is crucial to identify Chinese populations who are not only at risk, but who will benefit the most from prevention measures like smoking cessation, glucose and weight control, increased physical activity, and/ or high blood pressure or lipid-lowering treatments.

Tools to leverage risk factor modification strategies are available. Last year, researchers sought to develop and utilize a risk prediction equation that could be recalibrated for use in countries like China. Their study, which used a tool known as Globorisk, focused on identifying individuals at high risk of developing cardiovascular disease who are most likely to benefit from lifestyle changes or preventive drug treatment. The researchers suggested that "a next step would be to quantify the effects, on a population level, of introducing in these countries the Globorisk model combined with subsequent risk-based preventative management. These quantifications might further help ... convince decisionmakers across the world to decide on wide-scale introduction of prediction models and risk-based management for cardiovascular disease."[7]

Hyperlipidemia is a potential specific target for focus in China. Management of LDL-related ASCVD risk is an area where treatment gaps exist due in part to some confusion regarding guidelines with sometimes discordant or overlapping recommendations, as well as legitimate questions about whether data collected with regard to lipid treatment in Western studies is consistently applicable to Chinese patients. Given this, it is not all that surprising that statins are underprescribed for ASCVD prevention in China and questions exist about which guidelines to use, which lipoprotein measurement is preferred, and which drugs to prescribe.

Problematic is a perception of potential risks for adverse effects of statin therapy for hyperlipidemia, specific to the Chinese/Asian populations as a result of studies like HPS2-Thrive. This study found an increase in myopathy among Chinese patients who had been prescribed simvastatin compared to Europeans. ${ }^{[8]}$ The results led to a warning advising caution when prescribing simvastatin to Asian patients and instructions to use the lowest dose possible - a significant variation from the American College of Cardiology (ACC)/AHA Guidelines. ${ }^{[0]}$ Patient-related factors, including a preference among some for traditional Chinese medicine, a lack of understanding about long-term medication adherence, and even a limited understanding of ASCVD risk, are also problematic.

Until more definitive data are available, recognizing the areas where various guidelines are congruent is an important first step to managing ASCVD risk. Guidelines consistently recognize the etiologic importance of LDL-related ASCVD risk and all recommend that the intensity of therapy be matched to the risk of ASCVD events. All guidelines also recommend statins as first-line lipid-lowering therapy to reduce ASCVD events in moderate and high-risk patients.

As part of its missions to transform cardiovascular care and improve heart health around the world, the ACC, in collaboration with its China Chapter, the Chinese Society of Cardiology (CSC), and other stakeholders, is already working on a number of activities designed to reach Chinese physicians and patients where they live and work. For example, a unique "Train the Trainer" program implemented in 2013 focused on increasing awareness of atrial fibrillation treatment in China. Ten pilot centers were established throughout the country, which offered over 50 lectures and face-to-face exchanges in hospitals with top local and global experts in atrial fibrillation and embolic stroke prevention. To date, the program has reached over 4000 doctors across China.

More recently, the ACC and CSC launched a cardiovascular disease education and awareness program that will prepare Chinese physicians and hospital systems for a nationwide health care shift that supports heart disease prevention and optimal patient care. The ACC/CSC program includes a comprehensive webinar series targeted at hospitals throughout China. The first webinar, held in January 2016, reached 3400 doctors from 342 hospitals and was the largest one-time ACC educational program outside the United States. This partnership will also help develop topic-based interactive online education resources and tools for cardiologists and cardiac care team members delivered through WeChat—one of the most widely used social media platforms in China.

The College is also committed to helping China monitor national data on cardiovascular health to identify trends over time and track improvements in care. Integrated registries have the potential to be used as powerful tools for identifying education and quality improvement opportunities both globally and at country-specific levels. The ACC's National Cardiovascular Data Registry has made considerable contributions toward improving the quality of cardiac care in the United States, and there is tremendous potential internationally to use registries to inform clinical research, identify gaps in local care, and help providers develop best practices related to cardiovascular outcomes.

The ACC recently joined with the National Heart Centre Singapore (NHCS) to create a global diabetes registry that will provide insight into real-world treatment patterns in Asian patients, describe effectiveness and safety for key treatment classes and product types in more diverse populations, and will help quantify real-world treatment 
adherence. It will also provide information about major complications of diabetes and understanding of treatment outcomes in sociocultural subgroups. The joint effort will bring the NHCS Asian Diabetes Outcomes Registry, known as ADORE, into partnership with the Diabetes Collaborative Registry, to create the first global, cross-specialty clinical diabetes registry. ADORE will collect comprehensive data for type 2 diabetes treatment patterns and outcomes throughout Asia with a goal to create a network of sites with thousands of patients in China, South Korea, Taiwan, Singapore, Indonesia, Malaysia, Thailand, the Philippines, Hong Kong, India, Vietnam, and Sri Lanka.

The College has a number of other efforts underway to educate patients and clinicians about risk factors and evidence-based treatment. The College has been working to translate its CardioSmartonline patient-education platform and fact sheets into Mandarin. Additionally, select articles from the Journal of the American College of Cardiology (JACC): Interventions are also being translated into Mandarin to ensure clinicians can stay up-to-date on the growing field of interventional cardiology. "Hospitals from middle-sized to massive are performing 1000 to 13,000 interventional procedures per year," noted Spencer King, MD, MACC, Editor-in-Chief of JACC Interventions. "Laboratories are modern, and some run almost 24/7. Teaching, or level 3 hospitals, are the recipients of very large patient volumes with some caring for 10,000 outpatient visits per day. These are major urban centers, and I am sure rural China is vastly different, but one is struck by the size of these endeavors". ${ }^{10]}$ Volumes seen in many of these Chinese centers cannot be easily duplicated in the United States and Europe. The influence of the available data can potentially transform care worldwide and appropriately position China as a source of essential medical knowledge.

As the world's most populous country, China has the opportunity to not only curb the global cardiovascular epidemic domestically, but also to inform the international community in a way that affects patients in all countries. To date, most large Western trials have not enrolled sufficient numbers of Chinese patients and current evidence is often inadequate due to small sample size, low study quality, and short study duration. By participation in registries and focusing on standardized data collection, the Chinese experience can teach the rest of the world a great deal about how best to treat our patients worldwide. The medical "sleeping giant" of the $21^{\text {st }}$ century may well lie in China, and all of us look to learn from solid data forthcoming!

Looking to the future, there is momentum within China and at the global level to work together to decrease the burden of cardiovascular diseases. China and the United States, in particular have extraordinary opportunities to share information and best practices and to truly learn from each other-where we are the same and where we are different. In a 2015 editorial in the JACC, Editor-in-Chief Valentin Fuster, MD, PhD, MACC, said it best when he wrote: “This fight against the burden of cardiovascular disease, affecting all countries and local corners of the world, requires many physicians, specialists, and subspecialists." ${ }^{[11]}$ He stressed the need for concrete action, measurement, and follow-up, with our utmost goal to make a meaningful mark on cardiovascular disease around the world. China is a crucial international power in the fight against cardiovascular disease worldwide.

\section{Conflict of Interest}

None declared.

\section{References}

1. World Health Organization. Noncommunicable Diseases (NCD) Country Profiles 2014, July 2014, pg. 49, Available from: http://apps.who.int/ iris/bitstream/10665/128038/1/9789241507509_eng.pdf?ua=1. Accessed on February 12, 2016.

2. Bi Y, Jiang Y, He J, Xu Y, Wang L, Xu M, et al. Status of Cardiovascular Health in Chinese Adults. J Am Coll Cardiol 2015; 65: 1013-25.

3. Yang W. Changing characteristics of the type 2 diabetes epidemic of China and other Asian countries. J Diabetes Investig 2013; 4: 223-4.

4. Huang Y, Gao L, Xie X, Tan SC. Epidemiology of dyslipidemia in Chinese adults: meta-analysis of prevalence, awareness, treatment, and control. Popul Health Metr 2014; 12: 28.

5. He H, Yu Y, Li Y, Kou CG, Li B, Tao YC, et al. Dyslipidemia awareness, treatment, control and influence factors among adults in the Jilin province in China: a cross-sectional study. Lipids Health Dis 2014; 13: 122.

6. U.S. Department of Health and Human Services. The Health Consequences of Smoking-50 Years of Progress: A Report of the Surgeon General. Atlanta: U.S. Department of Health and Human Services, Centers for Disease Control and Prevention, National Center for Chronic Disease Prevention and Health Promotion, Office on Smoking and Health, 2014

7. Hajifathalian K, Ueda P, Lu Y, Woodward M, Ahmadvand A, AguilarSalinas CA, et al. A novel risk score to predict cardiovascular disease risk in national populations (Globorisk): a pooled analysis of prospective cohorts and health examination surveys. Lancet Diabetes Endocrinol 2015; 3: 339-55

8. HPS2-THRIVE Collaborative Group. HPS2-THRIVE randomized placebo-controlled trial in 25673 high-risk patients of ER niacin/ laropiprant: trial design, pre-specified muscle and liver outcomes, and reasons for stopping study treatment. Eur Heart J 2013; 34: 1279-91.

9. Stone NJ, Robinson JG, Lichtenstein AH, Bairey Merz CN, Blum CB, Eckel RH, et al. 2013 ACC/AHA Guideline on the Treatment of Blood Cholesterol to Reduce Atherosclerotic Cardiovascular Risk in Adults: A Report of the American College of Cardiology/American Heart Association Task Force on Practice Guidelines. J Am Coll Cardiol 2014; 63: 2889-934.

10. King SB 3rd. Lessons From China. J Am Coll CardiolIntv 2015; 8: 1911-2.

11. Fuster V. Global Burden of Cardiovascular Disease: Time to Implement Feasible Strategies and to Monitor Results. J Am Coll Cardiol 2014; 64: 520-2.

How to cite this article: Chazal RA. China may hold answers to addressing cardiovascular disease epidemic. J Transl Intern Med 2016; 4: 11-3. 